\title{
An Efficient Approach to Extrapolate the Data Falling in the Zone not Covered by Measurements in a Near-Field Spherical Facility
}

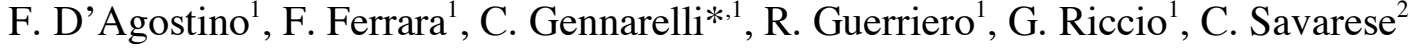

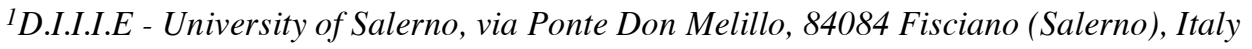 \\ ${ }^{2}$ DIT - University "Parthenope", via Medina 40, 80133 Naples, Italy
}

\begin{abstract}
In this paper, a new and efficient technique is developed to estimate the near-field data falling in the blind zone of a near-field spherical facility, i.e., the region not covered by the measurements. The proposed approach is based on the nonredundant sampling representations of the electromagnetic field and makes use of the singular value decomposition method for extrapolating the samples external to the measurement region. This allows a significant reduction of the truncation error occurring in the near field - far field transformation with spherical scanning. Numerical tests assessing the effectiveness of the technique are reported.
\end{abstract}

\section{INTRODUCTION}

The techniques for the reconstruction of antenna radiation patterns from near-field (NF) measurements have been widely investigated and used for applications ranging from cellular phone antennas to large phased arrays and complex multi-beam communication satellite antennas [1-3]. They have been proved to be efficient and attractive alternatives to conventional far-field (FF) and compact range measurements. Commonly, the measured NF data are transformed into FF patterns by using an expansion of the antenna field in terms of modes, namely, a complete set of solutions of the vector wave equation in the region outside the antenna. The type of modal expansion employed for representing the field is determined by the choice of the NF scanning surface. Thus, plane, cylindrical, or spherical waves are used when considering planar, cylindrical, and spherical surfaces, respectively. The orthogonality properties of the modes on such surfaces are then exploited to obtain the modal expansion coefficients, which allow the reconstruction of the antenna far field. The development and the spreading of NF-FF transformation techniques employing planar, cylindrical or spherical scanning systems are justified from the fact that each approach has its own particular advantages, depending on the antenna under test (AUT) and the measurement requirements. In particular, the NF-FF transformation technique with spherical scanning gives the full antenna pattern coverage, even though the data processing is considerably more complicated than that needed by transformations using planar and cylindrical NF facilities.

A considerable amount of work has been carried out in the past years for solving the problem of the FF reconstruction from NF data collected on a spherical scanning surface [4-11]. In this framework, a comprehensive book [8],

*Address correspondence to this author at the D.I.I.I.E - University of Salerno, via Ponte Don Melillo, 84084 Fisciano (Salerno), Italy;

E-mail: gennar@diiie.unisa.it which deals with the theoretical as well as the practical aspects of the spherical NF scanning, was published in 1988 by J.E. Hansen.

In [9], the standard NF-FF transformation with spherical scanning has been properly modified by taking into account the spatial bandlimitation properties of the electromagnetic (EM) fields [12]. In particular, the choice of the highest spherical wave has been rigorously fixed by the bandlimitation properties and the number of data on the parallels has resulted to be decreasing towards the poles. In the same paper, the non-redundant sampling representations of the EM field [13] have been applied to reduce in a significant way the number of needed NF data when considering antennas having one or two predominant dimensions. These results have been obtained by assuming the AUT as enclosed in a prolate or oblate ellipsoid, respectively, and by developing an optimal sampling interpolation (OSI) formula, which allows the reconstruction of the data required by the above mentioned NF-FF transformation. In $[10,11]$, the ideal probe assumption has been removed by proposing an efficient probe compensated NF-FF transformation with spherical scanning tailored for elongated or quasi-planar antennas.

Unfortunately, in most of spherical NF facilities, the presence of the AUT positioner prevents the possibility to carry out the measurements on the whole sphere. Therefore, there is a blind zone (shaded area in Fig. 1) wherein the NF data cannot be acquired. As a consequence, a truncation error affects the NF reconstruction in the zone close to the boundary of the measurement region. It is clear that the estimation of a proper number of samples falling in the blind zone becomes mandatory in order to improve the accuracy in such a zone and to obtain an accurate field reconstruction in the whole measurement region.

The goal of this paper is just the extrapolation of these samples. The estimation of such data (otherwise equal to zero in the application of the OSI algorithm) gives rise to a 
remarkable reduction of the truncation error in the periphery of the scanning region. Moreover, an acceptable reconstruction is attained in the whole blind zone or in a large part of it. The method is based on the aforementioned sampling representation and OSI expansion, and makes use of the singular value decomposition (SVD) algorithm [14] for determining the NF samples falling in the blind zone. For simplicity, the case of an ideal probe will be considered in the following, the extension to the case of a real probe being straightforward.

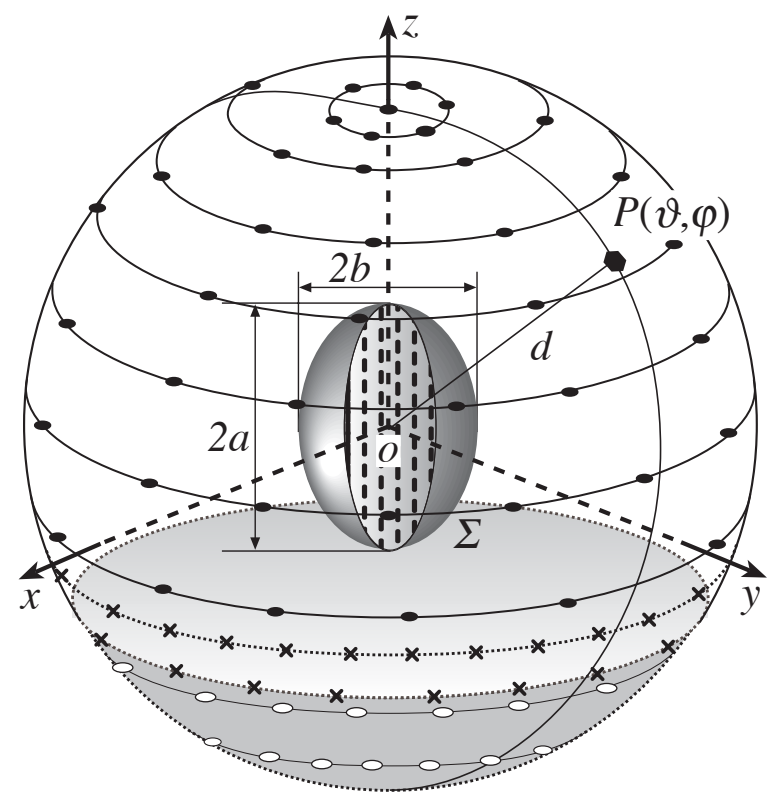

Fig. (1). Geometry of the problem.

\section{OPTIMAL SAMPLING REPRESENTATION OVER A SPHERE}

Let us consider the field radiated by an elongated AUT and observed on a spherical surface of radius $d$ in the NF region. An effective source modelling is obtained by choosing the surface $\Sigma$ (enclosing the AUT) coincident with the smallest prolate ellipsoid having major and minor semiaxes equal to $a$ and $b$, respectively (see Fig. 1). Since the sphere can be represented by meridians and parallels, in the following we deal with the field representation on an observation curve $C$ described by an analytical parameterization $\underline{r}=\underline{r}(\xi)$. According to [13], it is convenient to consider the "reduced electric field"

$\underline{F}(\xi)=\underline{E}(\xi) \mathrm{e}^{\mathrm{j} \gamma(\xi)}$

where $\gamma(\xi)$ is a proper phase function. The error, occurring when approximating $\underline{F}$ by a spatially bandlimited function, becomes negligible as the bandwidth exceeds a critical value $W_{\xi}$ [13]. Accordingly, such an error can be effectively controlled by choosing a bandwidth equal to $\chi^{\prime} W_{\xi}$, $\chi^{\prime}$ being an enlargement bandwidth factor (slightly greater than unity for electrically large antennas).

When the observation curve $C$ is a meridian, by adopting $W_{\xi}=\beta \ell^{\prime} / 2 \pi$, where $\beta$ is the free-space wavenumber and $\ell^{\prime}$ is the length of the ellipse

(obtained as intersection between the meridian plane and $\Sigma$ ), we get [13]:

$$
\begin{aligned}
& \gamma=\beta a\left[v \sqrt{\frac{v^{2}-1}{v^{2}-\varepsilon^{2}}}-E\left(\cos ^{-1} \sqrt{\frac{1-\varepsilon^{2}}{v^{2}-\varepsilon^{2}}} \mid \varepsilon^{2}\right)\right] \\
& \xi=\frac{\pi}{2}\left[1+\frac{E\left(\sin ^{-1} u \mid \varepsilon^{2}\right)}{E\left(\pi / 2 \mid \varepsilon^{2}\right)}\right]
\end{aligned}
$$

In the above equations, $E(\cdot \cdot \cdot)$ denotes the elliptic integral of second kind, $\varepsilon=f / a$ is the eccentricity of $C^{\prime}, 2 f$ is its focal distance and $u=\left(r_{1}-r_{2}\right) / 2 f, v=\left(r_{1}+r_{2}\right) / 2 a$ are the elliptic coordinates, $r_{1,2}$ being the distances from the observation point $P$ to the foci of $C^{\prime}$ (see Fig. 2). Relation (3) is valid for $\vartheta$ belonging to the range $[0, \pi / 2]$. The case when $\vartheta$ belongs to $[\pi / 2, \pi]$ can be easily handled by determining the value $\xi^{\prime}$ corresponding to the point specified by the angle $\pi-\vartheta$ and then putting $\xi=\pi-\xi^{\prime}$.

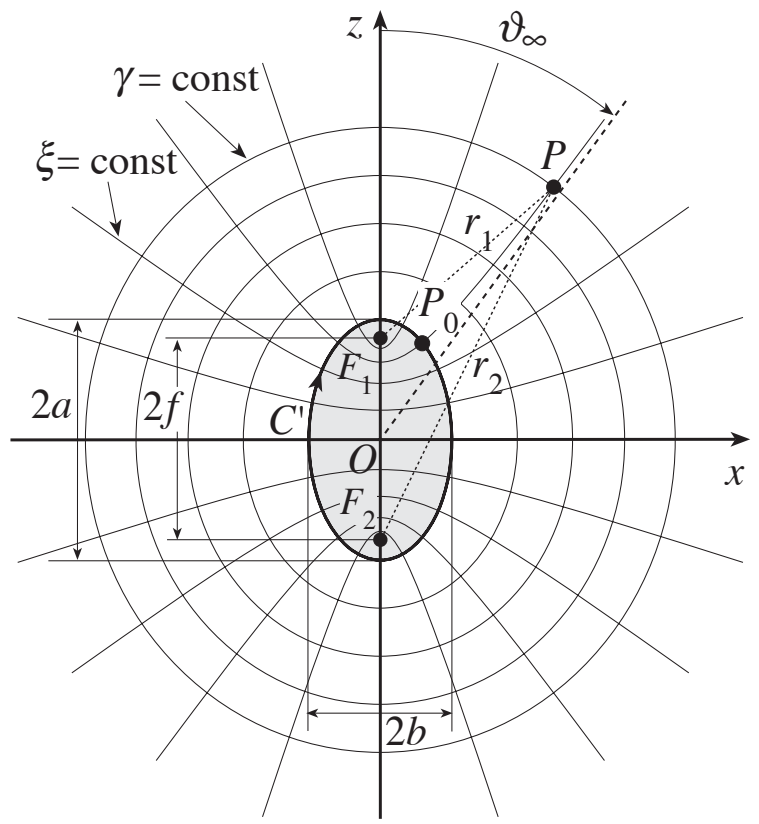

Fig. (2). Ellipsoidal source modelling: prolate case.

When $C$ is a parallel, due to the symmetry, the phase function is constant and it is convenient to use the azimuthal angle $\varphi$ as parameter [13]. The corresponding bandwidth is $W_{\varphi}(\xi)=\beta b \sin \vartheta_{\infty}(\xi)$, where $\vartheta_{\infty}=\sin ^{-1} u+\pi / 2$ is the polar angle of the asymptote to the hyperbola through $P$ (Fig. 2).

According to these results, a spherical component $F$ (along $\vartheta$ or $\varphi$ ) of the electric field at the point $P(\xi, \varphi)$ on the meridian fixed by $\varphi$ can be evaluated $[9,13]$ via the following OSI expansion:

$$
F(\xi, \varphi)=\sum_{n=n_{0}-q+1}^{n_{0}+q} F\left(\xi_{n}, \varphi\right) G\left(\xi, \xi_{n}, N, N^{\prime \prime}\right)
$$

where $F\left(\xi_{n}, \varphi\right)$ are the intermediate samples, i.e., the intersections of the considered meridian with the parallels 
fixed by the $\xi_{n}$ values, $2 q$ is the number of retained samples, $n_{0}=\operatorname{Int}(\xi / \Delta \xi)$ is the index of the sample nearest on the left to $P, \operatorname{Int}(x)$ giving the integer part of $x$, and

$$
G\left(\xi, \xi_{n}, N, N^{\prime \prime}\right)=D_{N^{\prime \prime}}\left(\xi-\xi_{n}\right) \Omega_{N}\left(\xi-\xi_{n}\right)
$$

In the above equation,

$$
\begin{aligned}
D_{N^{\prime \prime}}(\xi) & =\frac{\sin \left[\left(2 N^{\prime \prime}+1\right) \xi / 2\right]}{\left(2 N^{\prime \prime}+1\right) \sin (\xi / 2)} \\
\Omega_{N}(\xi) & =\frac{T_{N}\left[2\left(\cos (\xi / 2) / \cos \left(\xi_{0} / 2\right)\right)^{2}-1\right]}{T_{N}\left[2 / \cos ^{2}\left(\xi_{0} / 2\right)-1\right]}
\end{aligned}
$$

are the Dirichlet and Tschebyscheff Sampling functions, respectively, $T_{N}(\cdot)$ being the Tschebyscheff polynomial of degree $N$, and $\xi_{0}=q \Delta \xi$. Moreover,

$$
\begin{array}{ll}
\xi_{n}=n \Delta \xi=2 n \pi /\left(2 N^{\prime \prime}+1\right) ; & N^{\prime \prime}=\operatorname{Int}\left(\chi N^{\prime}\right)+1 \\
N^{\prime}=\operatorname{Int}\left(\chi^{\prime} W_{\xi}\right)+1 ; & N=N^{\prime \prime}-N^{\prime}
\end{array}
$$

wherein $\chi>1$ is an oversampling factor needed to control the truncation error.

The intermediate samples $F\left(\xi_{n}, \varphi\right)$ are given by:

$$
F\left(\xi_{n}, \varphi\right)=\sum_{m=m_{0}-p+1}^{m_{0}+p} F\left(\xi_{n}, \varphi_{m, n}\right) G\left(\varphi, \varphi_{m, n}, M_{n}, M_{n}^{\prime \prime}\right)
$$

where $F\left(\xi_{n}, \varphi_{m, n}\right)$ are the samples on the parallel fixed by $\xi_{n}, m_{0}=\operatorname{Int}\left(\varphi / \Delta \varphi_{n}\right)$, and $2 p$ is the number of retained samples. Moreover,

$$
\begin{aligned}
& \varphi_{m, n}=m \Delta \varphi_{n}=2 m \pi /\left(2 M_{n}^{\prime \prime}+1\right) \\
& M_{n}^{\prime \prime}=\operatorname{Int}\left(\chi M_{n}^{\prime}\right)+1 ; \quad M_{n}^{\prime}=\operatorname{Int}\left(\chi^{*} W_{\varphi_{n}}\right)+1 \\
& \chi^{*}(\xi)=1+\left(\chi^{\prime}-1\right)\left(\sin \vartheta_{\infty}(\xi)\right)^{-2 / 3} \\
& M_{n}=M_{n}^{\prime \prime}-M_{n}^{\prime}
\end{aligned}
$$

and the other symbols have the same meaning as in (4). Note that the variation of the azimuthal excess bandwidth factor $\chi^{*}$ with $\xi$ is required to ensure a bandlimitation error constant with respect to $\xi$ [9].

By properly matching the OSI expansions (4) and (10), it is so possible to reconstruct the tangential components of the electric field at any point on the scanning sphere and, in particular, at the points needed by the NF-FF transformation with spherical scanning [8] as modified in [9].

\section{ESTIMATION OF THE SAMPLES FALLING IN THE BLIND ZONE}

In the last years, the nonredundant sampling representations of EM fields have been widely employed for improving the existing NF-FF transformation techniques [9, 15-18] and for developing innovative ones $[19,20]$. In all the cases, the NF data required to perform the standard NF-FF transformations are recovered from a reduced number of samples by using proper OSI formulas. In fact, the main feature of these representations is to require a nonredundant and always finite number of samples also on an unbounded surface.

Obviously, the measurement region is inevitably truncated in the cylindrical and planar scannings, so that a truncation error affects the NF data recovery in the zones close to the boundary of the scanning region. As a consequence, the reconstruction results to be accurate in a zone smaller than the measurement one and this reflects in a decrease of the angular region wherein an accurate FF reconstruction is attained. Therefore, in order to obtain an accurate NF reconstruction in the whole scanning region, it is necessary to estimate a proper number of samples external to it. Such a problem has been tackled and solved, for instance, in the case of planar scannings [21-23]. In particular, the comparison of the extrapolation procedure based on the OSI expansions with that in [21] employing the cardinal series ones has shown that the former works better [22].

Now, although the measurement region in the spherical scanning should not be truncated, in the greater part of spherical NF facilities, the presence of the AUT positioner does not allow to collect the measurements on the whole sphere. As a consequence, a truncation error affects the NF reconstruction in the blind zone and in the region close to it. Accordingly, to get an accurate reconstruction in the whole measurement region and to improve the accuracy in the blind zone, it is very important, as already stated, to estimate a proper number of samples falling in it. And thus, the following extrapolation problem arises: estimation of a (spatially) bandlimited function outside the measurement region from the knowledge of its internal samples collected at a rate greater than the Nyquist one. As well-known, this is an ill-posed problem widely studied in literature with reference to the case of bandlimited signals known only in a finite time interval (see [22] for a selected bibliography).

Let us now focus on the estimation of the data lying in the blind zone $\left(\vartheta>\vartheta_{b}\right)$. The knowledge of the NF samples on the $K$ parallels at angles $\vartheta\left(\xi_{k}\right)$ uniformly distributed in ]$\left.\xi_{\bar{n}-1}, \xi\left(\vartheta_{b}\right)\right]$ is assumed, $\bar{n}$ being the index of the last regular parallel inside the scanning region (see Fig. 1). On each of these "extra parallels", the data are assumed known at the points specified by $\varphi_{m}=m \Delta \varphi_{\min }$, where $\Delta \varphi_{\min }$ is the spacing between the samples to be estimated on the first parallel external to the scanning region. When applying (4) on each of these points, which are aligned, only the samples falling in the blind zone are unknown, whereas the others can be easily determined via the OSI expansion (10). In order to exploit the information available on the other side of the meridian beyond the south pole, it is convenient that $\xi_{k}$ and $\xi_{n}$ can assume values greater than $\pi$. Accordingly, 
when $\left.\left.\quad \xi_{k} \in\right] \xi_{\bar{n}-1}, \xi\left(\vartheta_{b}\right)\right] \quad$ and $\quad q>\bar{n}-n_{0}+2 n_{b}$ ( $n_{b}=N^{\prime \prime}-\bar{n}$ being the number of parallels lying in the blind zone), it results:

$$
\begin{gathered}
\sum_{n=\bar{n}+1}^{\bar{n}+2 n_{b}} F\left(\xi_{n}, \varphi_{m}\right) G\left(\xi_{k}, \xi_{n}, N, N^{\prime \prime}\right)=F\left(\xi_{k}, \varphi_{m}\right)+ \\
-\sum_{n=n_{0}-q+1}^{\bar{n}} F\left(\xi_{n}, \varphi_{m}\right) G\left(\xi_{k}, \xi_{n}, N, N^{\prime \prime}\right)+ \\
-\sum_{n=\bar{n}+2 n_{b}+1}^{n_{0}+q} F\left(\xi_{n}, \varphi_{m}\right) G\left(\xi_{k}, \xi_{n}, N, N^{\prime \prime}\right) \\
k=1, \ldots, K
\end{gathered}
$$

Whereas, when $\xi_{k} \in\left[2 \pi-\xi\left(\vartheta_{b}\right), 2 \pi-\xi_{\bar{n}-1}[\quad\right.$ and $q>n_{0}-\bar{n}$, we get:

$$
\begin{gathered}
\sum_{n=\bar{n}+1}^{\bar{n}+2 n_{b}} F\left(\xi_{n}, \varphi_{m}\right) G\left(\xi_{k}, \xi_{n}, N, N^{\prime \prime}\right)=F\left(\xi_{k}, \varphi_{m}\right)+ \\
-\sum_{n=\bar{n}+2 n_{b}+1}^{n_{0}+q} F\left(\xi_{n}, \varphi_{m}\right) G\left(\xi_{k}, \xi_{n}, N, N^{\prime \prime}\right)+ \\
-\sum_{n=n_{0}-q+1}^{\bar{n}} F\left(\xi_{n}, \varphi_{m}\right) G\left(\xi_{k}, \xi_{n}, N, N^{\prime \prime}\right) \\
k=K+1, \ldots, 2 K
\end{gathered}
$$

The overdetermined linear equation system (15) and (16) can be rewritten in matrix form as

$\underline{\underline{A}} \underline{x}=\underline{b}$

where $\underline{x}$ is the sequence of the unknown samples $F\left(\xi_{n}, \varphi_{m}\right)$, with $n=\bar{n}+1, \ldots, \bar{n}+2 n_{b}, \underline{b}$ is the sequence of the known terms (those on the right hand side in (15) and (16)), and $\underline{A}$ is the $2 K \times 2 n_{b}$ matrix, whose elements are given by the weight functions in the considered OSI expansion:

$A_{k n}=G\left(\xi_{k}, \xi_{n}, N, N^{\prime \prime}\right)$

The overdetermined system (17) is ill-conditioned due to the presence of the bandlimitation and measurement errors, and the use of the SVD technique allows one to find the best approximation to the solution in the least squares sense. In particular, as already done in [22] with reference to the plane-polar scanning case, it is convenient to employ the truncated version of SVD algorithm in order to improve the accuracy of the solution $[14,24]$.

When the number of samples falling in the blind zone grows up, there is no gain to employ the data beyond the south pole. Moreover, it is convenient to reduce the number of unknowns, cutting away those corresponding to the farther sampling points, since only a small number of outside samples can be reliably recovered [22]. Accordingly, in such a case, $\xi_{k}$ and $\xi_{n}$ no longer assume values greater than $\pi$, and the overdetermined linear equation system to be solved becomes:

$$
\begin{gathered}
\sum_{n=\bar{n}+1}^{\bar{n}+\bar{q}} F\left(\xi_{n}, \varphi_{m}\right) G\left(\xi_{k}, \xi_{n}, N, N^{\prime \prime}\right)=F\left(\xi_{k}, \varphi_{m}\right)+ \\
-\sum_{n=n_{0}-q+1}^{\bar{n}} F\left(\xi_{n}, \varphi_{m}\right) G\left(\xi_{k}, \xi_{n}, N, N^{\prime \prime}\right) \\
k=1, \ldots, K
\end{gathered}
$$

where it is assumed that $\bar{q} \leq n_{b}$ and $\bar{q} \leq q$.

\section{NF-FF TRANSFORMATION WITH SPHERICAL SCANNING}

In the following, the key steps of the classical NF-FF transformation with spherical scanning [8] as modified in [9] are reported for reader's convenience.

As well known, the tangential electric field in the FF region may be written by means of the truncated spherical wave expansion:

$$
\begin{aligned}
& \underline{E}_{t}(R \rightarrow \infty, \Theta, \Phi)=\frac{\mathrm{e}^{-\mathrm{j} \beta R}}{R} .
\end{aligned}
$$

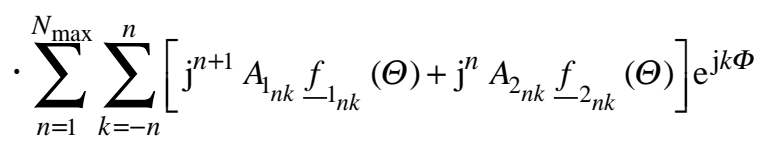

wherein the spherical coordinate system $(R, \Theta, \Phi)$ has been adopted for denoting the FF observation point.

In the classical approach, the choice of the highest spherical wave is usually determined according to the following rule-of-thumb:

$$
N_{\text {max }}=\operatorname{Int}(\beta a)+10
$$

where $a$ is the radius of the smallest sphere enclosing the AUT. In the here described approach, the highest spherical harmonic is rigorously fixed by the aforementioned bandlimitation properties of the radiated EM fields [12]. Accordingly:

$N_{\max }=\operatorname{Int}\left(\chi^{\prime} \beta a\right)+1$

The vectorial functions $\underline{f}_{1,2}(\Theta)$ are given by:

$$
\begin{aligned}
& \underline{f}_{1_{n k}}(\Theta)=\left(\frac{-k}{|k|}\right)^{k} \frac{1}{\sqrt{2 \pi n(n+1)}} \cdot \\
& \cdot\left[\frac{\mathrm{j} k}{\sin \Theta} \bar{P}_{n}^{|k|}(\cos \Theta) \hat{\Theta}+\frac{\mathrm{d}}{\mathrm{d} \Theta} \bar{P}_{n}^{|k|}(\cos \Theta) \hat{\Phi}\right] \\
& \underline{f}_{n k}(\Theta)=\left(\frac{-k}{|k|}\right)^{k} \frac{1}{\sqrt{2 \pi n(n+1)}} .
\end{aligned}
$$


$\cdot\left[\frac{\mathrm{d}}{\mathrm{d} \Theta} \bar{P}_{n}^{|k|}(\cos \Theta) \hat{\Theta}+\frac{\mathrm{j} k}{\sin \Theta} \bar{P}_{n}^{|k|}(\cos \Theta) \hat{\Phi}\right]$

where $\bar{P}_{n}^{|k|}(\cos \Theta)$ are the normalized associated Legendre functions as defined by Belousov in [25]. The expansion coefficients in (20) can be evaluated from the knowledge of the tangential electric field on the measurement sphere:

$$
\begin{aligned}
& A_{1,2 n k}=\frac{1}{\beta g_{1,2 n}(\beta d)} \cdot \\
& \cdot \int_{0}^{\pi} \int_{-\pi}^{\pi} \underline{E}_{t}(d, \vartheta, \varphi) \cdot \underline{f}_{1,2 n k}^{*}(\vartheta) \mathrm{e}^{-\mathrm{j} k \varphi} \sin \vartheta \mathrm{d} \varphi \mathrm{d} \vartheta
\end{aligned}
$$

in which (.) indicates the inner product, $\left(^{*}\right)$ denotes the complex conjugate, and

$g_{1_{n}}(x)=h_{n}^{(2)}(x)$

$g_{2_{n}}(x)=\frac{1}{x} \frac{\mathrm{d}}{\mathrm{d} x}\left[x h_{n}^{(2)}(x)\right]$

$h_{n}^{(2)}(x)$ being the spherical Hankel function of second kind and order $n$.

As shown in [9], the integration over $\varphi$ in relation (25) can be efficiently performed by expanding the tangential electric field components in Fourier series with respect to $\varphi$, namely,

$$
E_{\vartheta, \varphi}(d, \vartheta, \varphi)=\sum_{m=-M_{\varphi}}^{M_{\varphi}} G_{\vartheta, \varphi m}(\vartheta) \mathrm{e}^{\mathrm{j} m \varphi}
$$

where the number of terms is rigorously determined by the azimuthal bandwidth of the AUT, i.e., $M_{\varphi}=\operatorname{Int}\left(\chi^{*} \beta a \sin \vartheta\right)+1$.

In a quite similar way, the remaining integration over $\vartheta$ can be efficiently carried out by expanding the components of $\underline{G}_{k}(\vartheta)$ and $\underline{f}_{1,2_{n k}}(\vartheta)$ in Fourier series. Accordingly, we

$$
\begin{aligned}
& A_{1,2 n k}=\frac{2 \pi}{\beta g_{1,2 n}(\beta d)} \cdot \\
& \cdot \sum_{\ell=-N_{\max }}^{N_{\max }} \sum_{i=-N_{\max }}^{N_{\max }} \underline{G}_{k \ell} \cdot \underline{f}_{1,2_{n k i}}^{*} \int_{0}^{\pi} \mathrm{e}^{\mathrm{j}(\ell-i) \vartheta} \sin \vartheta \mathrm{d} \vartheta
\end{aligned}
$$

It is worthy to note that, in order to evaluate the Fourier series coefficients of the components of $\underline{G}_{k}(\vartheta)$ and $f_{1,2 n k}(\vartheta)$, it is necessary to extend these components from $[0, \pi]$ on the range $[-\pi, \pi]$. This can be easily done by taking into account that:

i) the components of $\underline{f}_{1,2 n k}(\vartheta)$ are even when $k$ is odd, and vice versa;

ii) the components of $\underline{G}_{k}(\vartheta)$ have the same parity as those of $\underline{f}_{1,2 n k}(\vartheta)$.
To take advantage of the numerical efficiency of the standard FFT algorithm, the number of NF parallels to be considered in the NF-FF transformation and the number of samples on them must be the first power of two greater or equal to $N_{\max }$ and $2 M_{\varphi}$, respectively.

From a computational viewpoint, it is convenient to invert the summation order in (20), so that it can be rewritten in the form:

$$
\begin{aligned}
& \underline{E}_{t}(R \rightarrow \infty, \Theta, \Phi)=\frac{\mathrm{e}^{-\mathrm{j} \beta R}}{R} . \\
& \cdot \sum_{k=-M_{\varphi}}^{M_{\varphi}} \sum_{\substack{n=|k| \\
n \neq 0}}^{N_{\max }}\left[\mathrm{j}^{n+1} A_{1_{n k}} \underline{f}_{1_{n k}}(\Theta)+\mathrm{j}^{n} A_{2_{n k}} \underline{f}_{2_{n k}}(\Theta)\right] \mathrm{e}^{\mathrm{j} k \Phi}
\end{aligned}
$$

which allows an efficient evaluation of the antenna far field at the considered elevation angle $\Theta$ by performing the summation via FFT.

It is worthy to note that, for computer time saving, it is convenient to apply the described FF reconstruction process to evaluate only the strictly needed FF samples. This allows one to recover accurately the FF components everywhere, by applying an OSI expansion properly modified to deal with an even number of samples on the FF parallels [9].

\section{NUMERICAL RESULTS}

Two sets of simulations are reported in the following. The former is relevant to a case occurring when the number of parallels falling in the blind region allows one to take advantage of the information available on the other side of the meridian. Whereas, the latter refers to a case when such a number is increased so much that it is no more possible to estimate all the unknown samples and, as a consequence, there is no gain to employ the data beyond the south pole.

In the first case, the numerical tests refer to a uniform planar array of $0.7 \lambda$ spaced elementary Huygens sources polarized along the $z$ axis and lying in an elliptical zone on the plane $y=0$, with major and minor semi-axes equal to $9 \lambda$ and $4 \lambda$, respectively ( $\lambda$ being the wavelength). The radius $d$ of the scanning sphere is $20 \lambda$. It is assumed that, due to the presence of the AUT positioner, the scanning region is limited to the angle $\vartheta_{b}=165^{\circ}$. According to the sampling representation, the last regular parallel inside the scanning region is at $\vartheta=162.8^{\circ}$, the number $n_{b}$ of parallels lying in the blind zone is 4 , and $K=6$ extra parallels have been considered, thus the computational effort to perform the SVD is negligible. Moreover, $q=19$ has been assumed in (15) and (16), whereas $p=13$ has been adopted in (10) for obtaining the involved known samples. Note that, as already stated, the $\vartheta$ values specifying the extra parallels have been chosen uniformly spaced in $\xi$ and belong to the $\vartheta$ range ] $\left.159.4^{\circ}, 165^{\circ}\right],\left(159.4^{\circ}\right.$ corresponds to the penultimate "regular parallel"). Fig. 3 shows the amplitude of the NF 


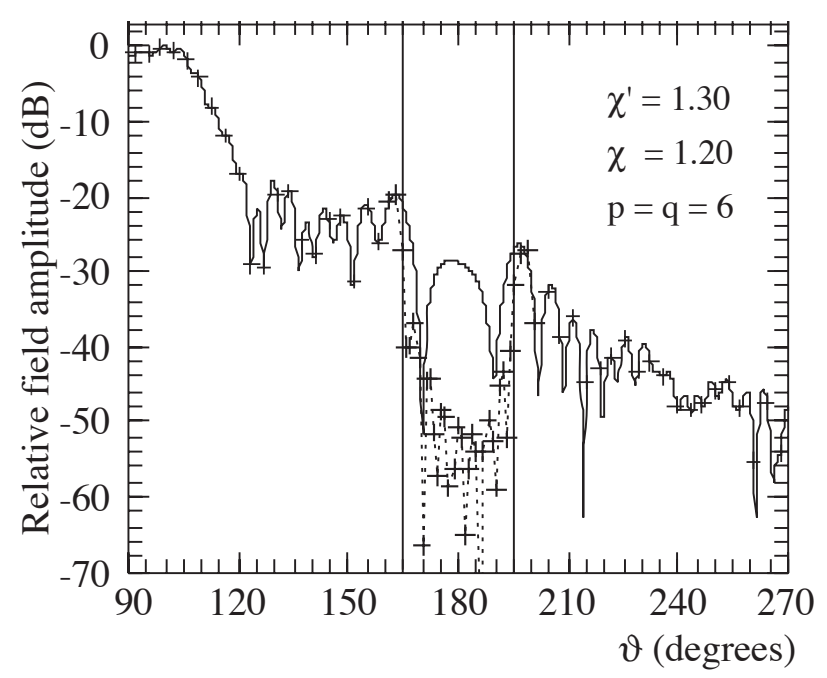

Fig. (3). Array 1. Amplitude of the NF $\vartheta$-component at $\varphi=90^{\circ}$. Solid line: exact. Crosses: reconstructed without estimated samples.

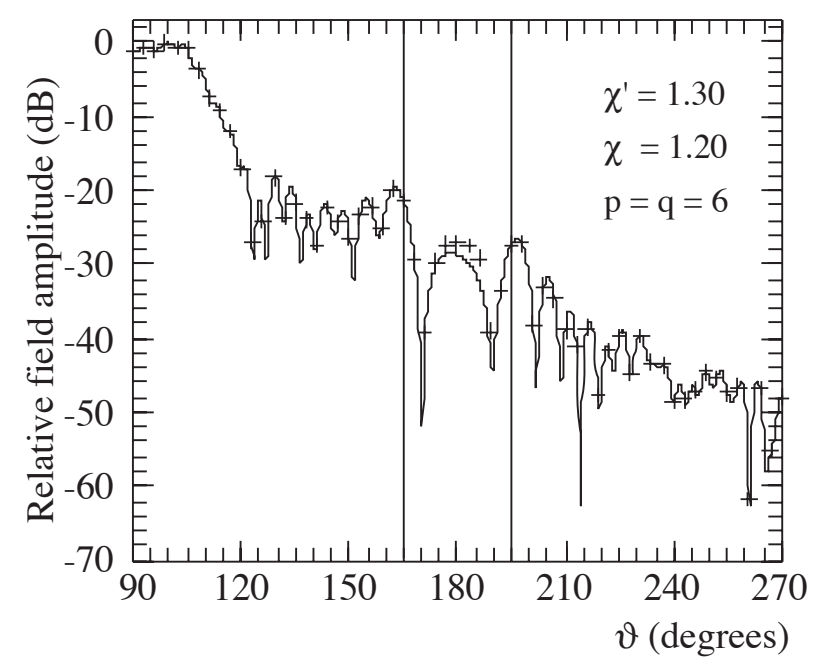

Fig. (4). Array 1. Amplitude of the NF $\vartheta$-component at $\varphi=90^{\circ}$. Solid line: exact. Crosses: reconstructed with estimated samples.

$\vartheta$-component (the most significant one) on the meridian at $\varphi=90^{\circ}$. It has been reconstructed without using the extrapolation process. As can be seen in Fig. 4, by using the here proposed estimation procedure, the reconstruction is very accurate not only in the whole measurement region, but also in an extended zone outside it, which practically covers the whole blind region. The same comments hold also for the following Figs. 5 and $\mathbf{6}$, which refer to the meridian at $\varphi=45^{\circ}$. To assess the effectiveness of the approach in a more quantitative way, the maximum and mean-square reconstruction errors have been evaluated by comparing (in the measurement zone) the exact values and those reconstructed with and without the estimated outside samples. Fig. 7 shows such errors, normalized to the maximum field value on the sphere, versus the $p=q$ values used in the final interpolation step. As can be seen, the errors evaluated by taking into account the estimated samples decrease until very low levels are reached, whereas those obtained without considering them saturate quickly. The proposed technique has been applied to recover the data needed for the classical NF-FF transformation with spherical scanning as modified in [9]. Figs. 8 and $\mathbf{9}$ report the AUT pattern in the E-plane, reconstructed by means of the NF-FF transformation without and with the estimated outside samples, respectively. As can be seen, the FF reconstruction obtained by considering the estimated samples is accurate in a remarkably wider angular range. It is useful to note that the number of the acquired NF data is 4385 (the extra data are 212), less than that (32514) needed by the NF-FF transformation [8].

The latter set of simulations refers to an electrically larger antenna and is representative of the case when it is not convenient to employ the data beyond the south pole. The radius $d$ of the scanning sphere is now $35 \lambda$, and the AUT is a uniform planar array of $0.6 \lambda$ spaced elementary Huygens sources polarized along the $z$ axis and lying in an elliptical zone on the plane $y=0$, with major and minor semi-axes equal to $20 \lambda$ and $6 \lambda$. In such a case, $\vartheta_{b}=164^{\circ}, n_{b}=7$, and $K=9$ extra parallels have been considered. Moreover, $\bar{q}=n_{b}$ and $q=19$ have been assumed in (19), whereas $p=13$ has been adopted in (10) for obtaining the involved known samples. Figs. $\mathbf{1 0}$ and $\mathbf{1 1}$ show the amplitude of the NF $\vartheta$-component on the meridian at $\varphi=90^{\circ}$, reconstructed without and with the estimation of the samples falling in the blind region. As can be seen, the reconstruction obtained by taking into account the estimated samples is very accurate also in a portion of the zone not covered by the measurements. The effectiveness of the approach is also confirmed by the values of the maximum and mean-square reconstruction errors reported in Fig. 12. As in the previous simulation, they have been obtained by comparing (in the measurement zone) the exact values and those reconstructed with and without the estimated outside samples. As expected, the errors evaluated by taking into account the estimated samples decrease until very low levels are reached. The algorithm stability has been investigated by corrupting the exact samples with random errors, which simulate a background noise (bounded to $\Delta a$ in amplitude and with arbitrary phase) and an uncertainty on the data of $\pm \Delta a_{r}$ in amplitude and $\pm \Delta \alpha$ in phase. As shown in Fig. 13, the technique gives satisfactory results also in presence of errors. Note that, in such a case, we have adopted $q=13$ and $p=9$ in the extrapolation procedure to reduce the propagation of errors from high to low field regions. Figs. 14 and 15 report the FF pattern in the E-plane, recovered without and with the estimation of the samples falling in the blind region. In order to improve the readability of the results, each figure has been split into two parts. In the former, $\Theta$ ranges from $90^{\circ}$ to $180^{\circ}$, whereas the latter is relevant to the $\Theta$-range $\left[180^{\circ}\right.$, $270^{\circ}$ ]. Also in such a case, the FF reconstruction obtained by taking into account the estimated samples is accurate in a significantly wider angular range. 


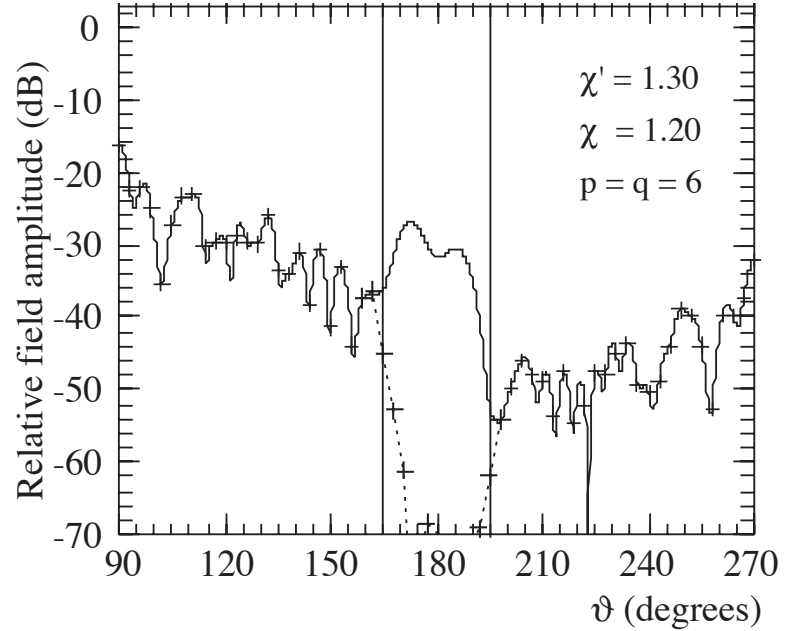

Fig. (5). Array 1. Amplitude of the NF $\vartheta$-component at $\varphi=45^{\circ}$. Solid line: exact. Crosses: reconstructed without estimated samples.

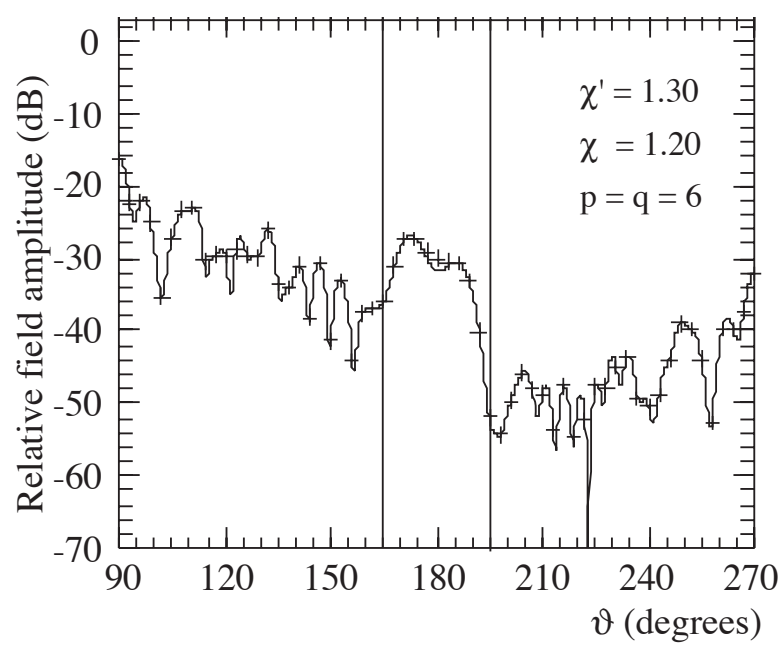

Fig. (6). Array 1. Amplitude of the NF $\vartheta$-component at $\varphi=45^{\circ}$. Solid line: exact. Crosses: reconstructed with estimated samples.

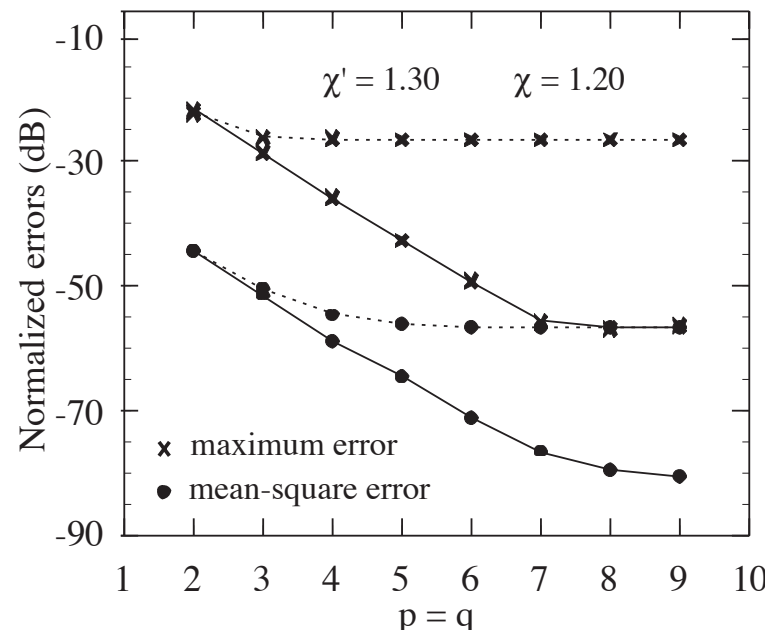

Fig. (7). Array 1. Reconstruction errors of the NF $\vartheta$-component. Dashed line: without estimated samples. Solid line: with estimated samples.

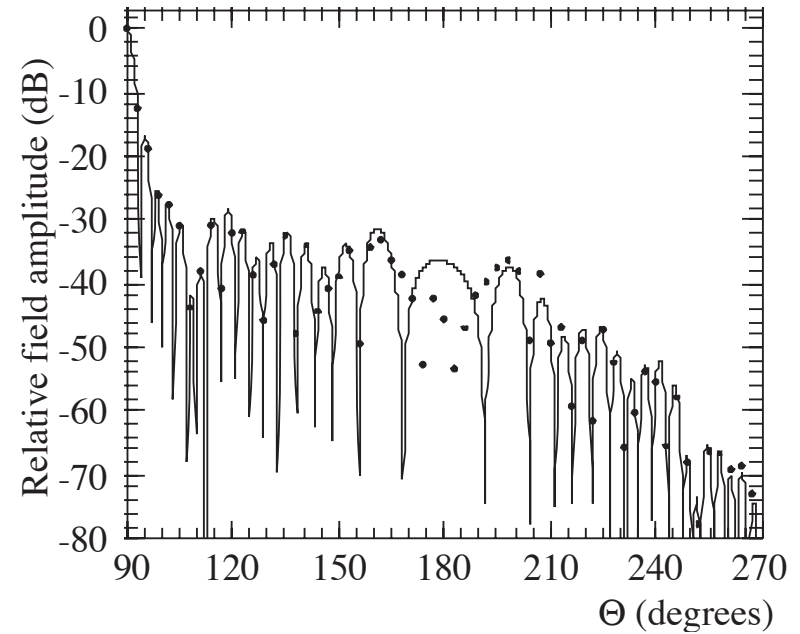

Fig. (8). Array 1. FF pattern in the E-plane. Solid line: exact. Dots: reconstructed from NF data without estimated samples.

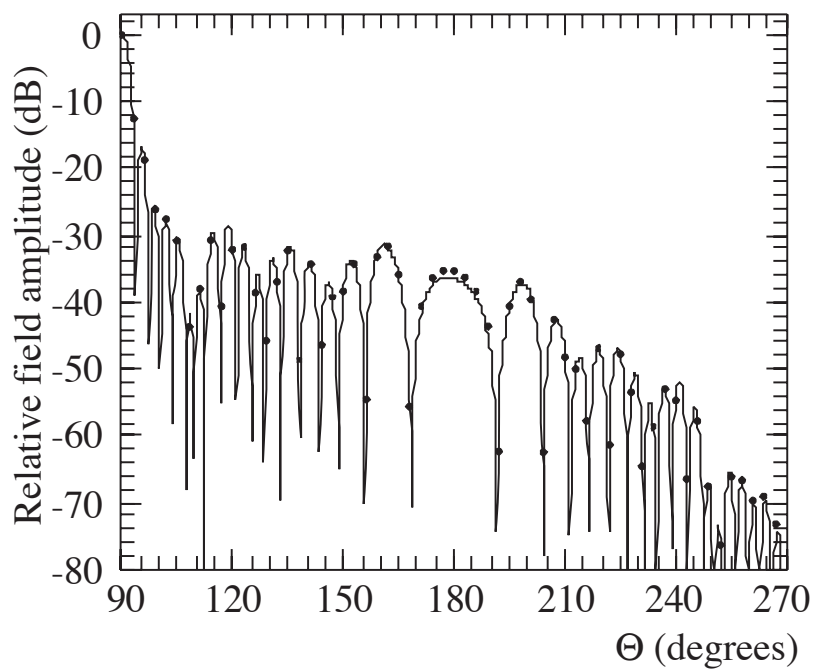

Fig. (9). Array 1. FF pattern in the E-plane. Solid line: exact. Dots: reconstructed from NF data with estimated samples.

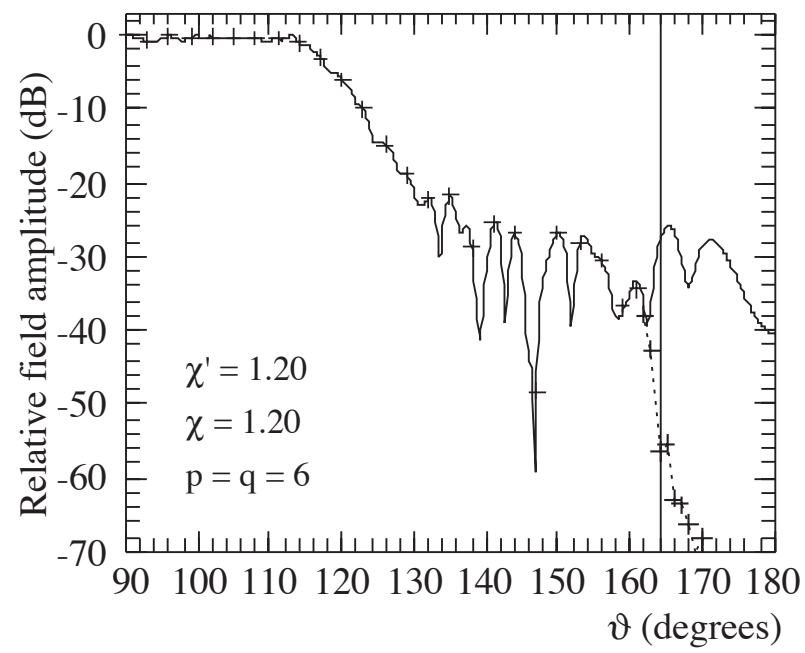

Fig. (10). Array 2. Amplitude of the NF $\vartheta$-component at $\varphi=90^{\circ}$. Solid line: exact. Crosses: reconstructed without estimated samples. 


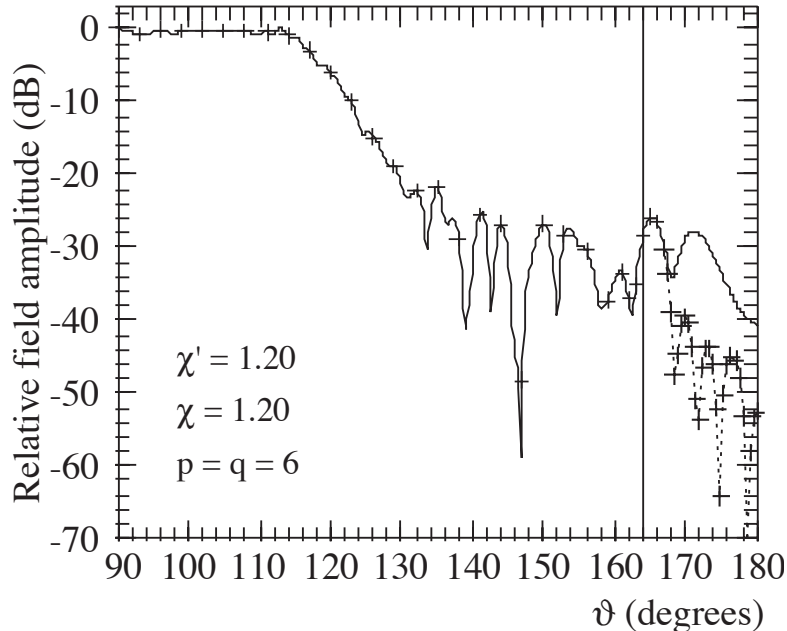

Fig. (11). Array 2. Amplitude of the NF $\vartheta$-component at $\varphi=90^{\circ}$. Solid line: exact. Crosses: reconstructed with estimated samples.

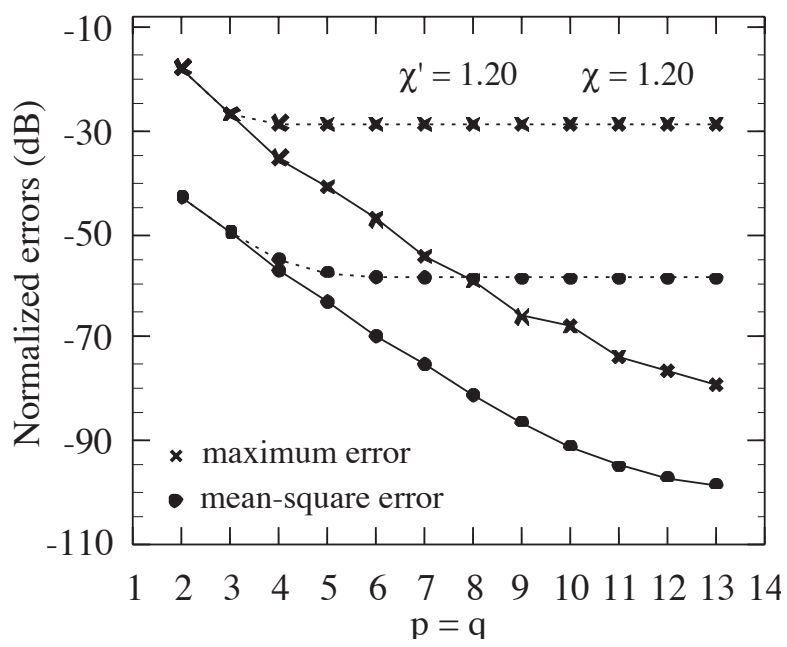

Fig. (12). Array 2. Reconstruction errors of the NF $\vartheta$-component. Dashed line: without estimated samples. Solid line: with estimated samples.

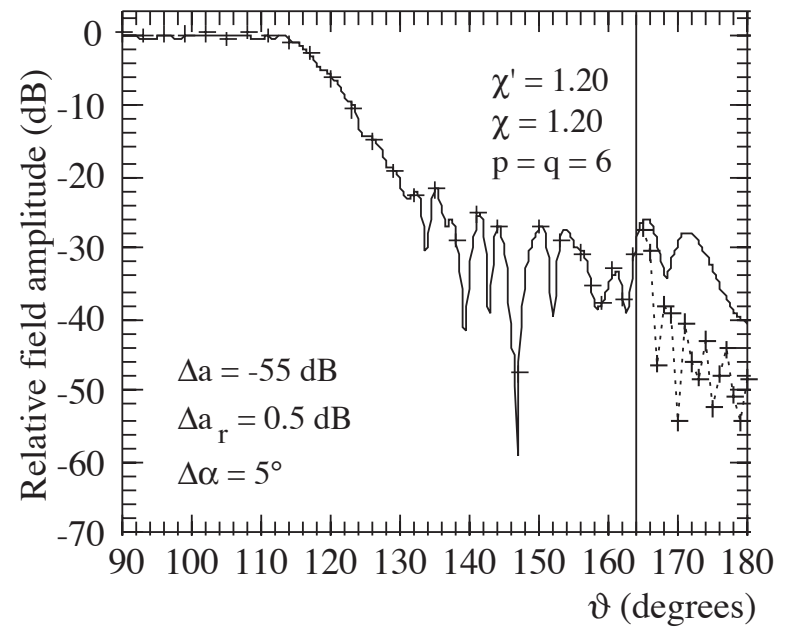

Fig. (13). Array 2. Amplitude of the NF $\vartheta$-component at $\varphi=90^{\circ}$. Solid line: exact. Crosses: reconstructed from error affected data with estimated samples.

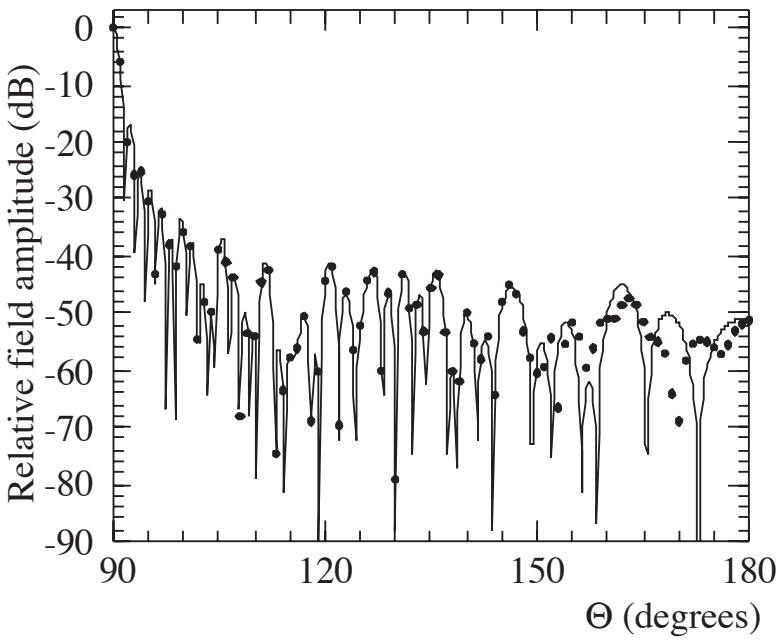

Fig. (14a). Array 2. FF pattern in the E-plane $\left(\Theta\right.$ ranging from $90^{\circ}$ to $\left.180^{\circ}\right)$. Solid line: exact. Dots: reconstructed from NF data without estimated samples.

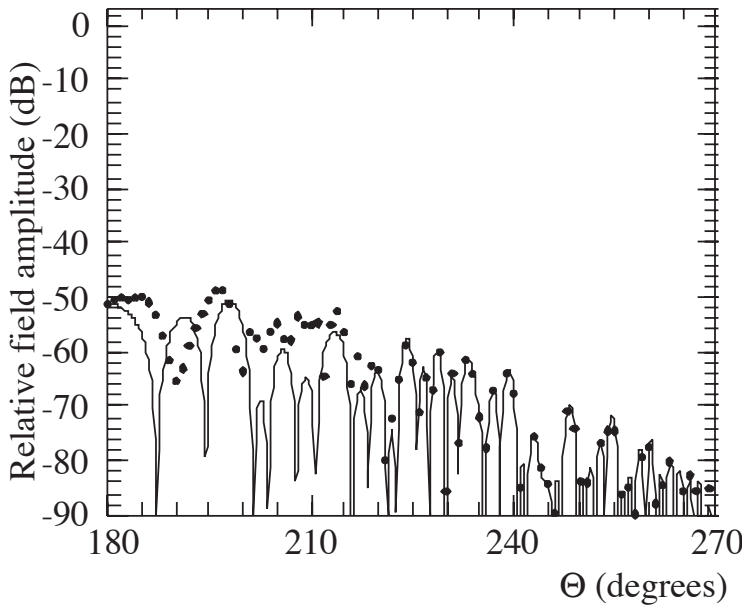

Fig. (14b). Array 2. FF pattern in the E-plane ( $\Theta$ ranging from $180^{\circ}$ to $270^{\circ}$ ). Solid line: exact. Dots: reconstructed from NF data without estimated samples.

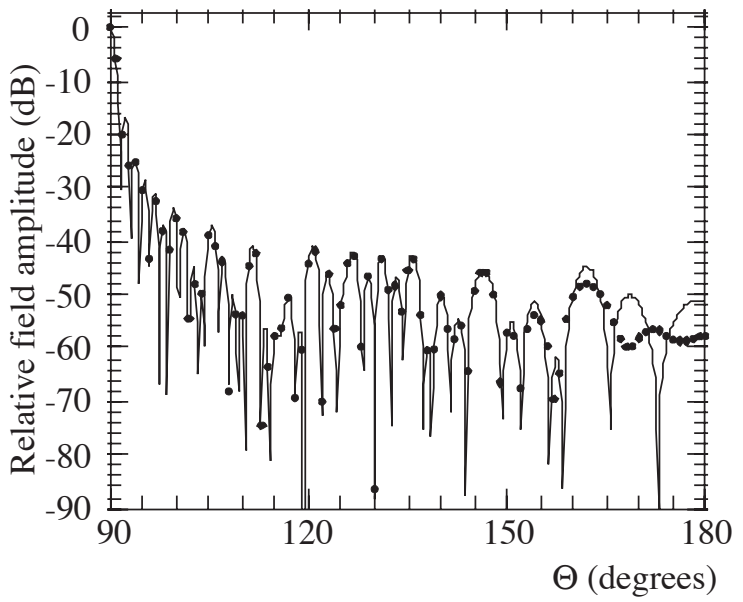

Fig. (15a). Array 2. FF pattern in the E-plane ( $\Theta$ ranging from $90^{\circ}$ to $180^{\circ}$ ). Solid line: exact. Dots: reconstructed from NF data with estimated samples. 


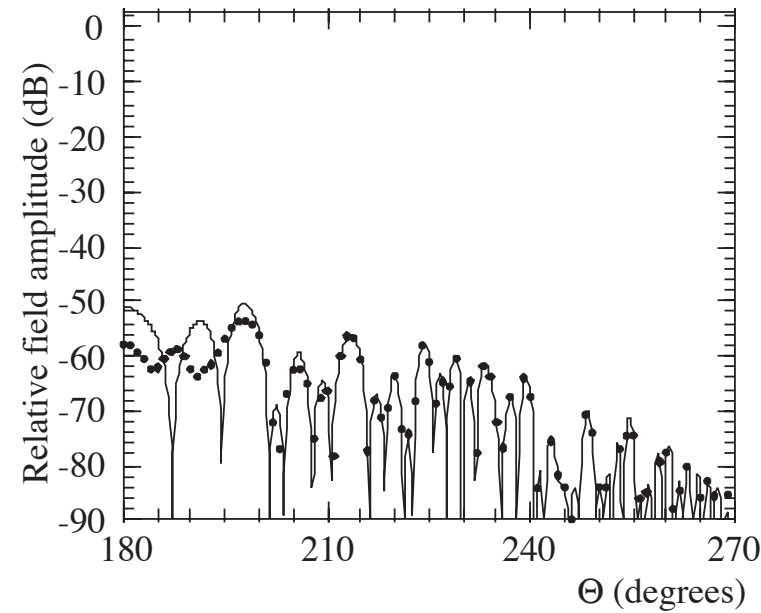

Fig. (15b). Array 2. FF pattern in the E-plane $\left(\Theta\right.$ ranging from $180^{\circ}$ to $270^{\circ}$ ). Solid line: exact. Dots: reconstructed from NF data with estimated samples.

\section{REFERENCES}

[1] "Special Issue on near-field scanning techniques," IEEE Trans. Antennas Propagat., vol. AP-36, pp. 727-901, June 1988.

[2] A.D. Yaghjian, "An overview of near-field antenna measurements," IEEE Trans. Antennas Propagat., vol. AP-34, pp. 30-45, January 1986.

[3] C. Gennarelli, G. Riccio, F. D'Agostino, and F. Ferrara, Near-field - far-field transformation techniques, vol.1, CUES, Salerno, Italy, November 2004.

[4] P.F. Wacker, "Non-planar near-field measurements: Spherical scanning," NBSIR 75-809, Boulder, CO, 1975.

[5] F.H. Larsen, "Probe-corrected spherical near-field antenna measurements," Ph.D. dissertation, Tech. Univ. of Denmark, Rep. LD36, 1980 .

[6] A.D. Yaghjian and R.C. Wittmann, "The receiving antenna as a linear differential operator: application to spherical near-field measurements," IEEE Trans. Antennas Propagat., vol. AP-33, pp. 1175-1185, November 1985.

[7] T.B. Hansen, "Formulation of spherical near-field scanning for electromagnetic fields in the time domain," IEEE Trans. Antennas Propagat., vol. 45, pp. 620-630, April 1997.

[8] J.E. Hansen, ed., Spherical Near-Field Antenna Measurements, IEE Electromagnetic Waves Series, Peter Peregrinus, London, UK, 1988.

[9] O.M. Bucci, F. D'Agostino, C. Gennarelli, G. Riccio, and C. Savarese, "Data reduction in the NF-FF transformation technique with spherical scanning," J. Electromagn. Waves Appl., vol.15, pp.755-775, 2001.

[10] F. D'Agostino, C. Gennarelli, G. Riccio, and C. Savarese, "A nonredundant probe compensated NF-FF transformation with spherical scanning," Proc. of 2003 IEEE AP-S Int. Symp., Columbus, Ohio, USA, June 2003.
[11] A. Arena, F. D'Agostino, C. Gennarelli, and G. Riccio, "Probe compensated NF-FF transformation with spherical scanning from a minimum number of data," Atti della Fondazione G.Ronchi, vol. 59, pp. 312-326, 2004.

[12] O.M. Bucci and G. Franceschetti, "On the spatial bandwidth of scattered fields," IEEE Trans. Antennas Propagat., vol. AP-35, pp. 1445-1455, December 1987.

[13] O.M. Bucci, C. Gennarelli, and C. Savarese, "Representation of electromagnetic fields over arbitrary surfaces by a finite and nonredundant number of samples," IEEE Trans. Antennas Propagat., vol. 46, pp. 351-359, March 1998.

[14] G. H. Golub and C. F. Van Loan, Matrix computations. Baltimore: J.Hopkins University Press, 1996.

[15] O.M. Bucci, F. D’Agostino, C. Gennarelli, G. Riccio, and C. Savarese, "NF-FF transformation with plane-polar scanning: ellipsoidal modelling of the antenna," Automatika, vol. 41, pp. 159164, 2000.

[16] F. D'Agostino, C. Gennarelli, G. Riccio, and C. Savarese, "Data reduction in the NF-FF transformation with bi-polar scanning," Microw. Opt. Technol. Lett., vol. 36, pp. 32-36, January 2003.

[17] O.M. Bucci, C. Gennarelli, G. Riccio, and C. Savarese, "NF-FF transformation with cylindrical scanning: an effective technique for elongated antennas," IEE Proc. - Microw., Antennas Propagat., vol. 145, pp. 369-374, October 1998.

[18] F. D'Agostino, F. Ferrara, C. Gennarelli, G. Riccio, and C. Savarese, "NF-FF transformation with cylindrical scanning from a minimum number of data," Microw. Opt. Technol. Lett., vol. 35, pp. 264-270, November 2002.

[19] F. Ferrara, C. Gennarelli, R. Guerriero, G. Riccio, and C. Savarese, "An efficient near-field to far-field transformation using the planar wide-mesh scanning," J. Electromagn. Waves Appl., vol. 21, pp. 341-357, 2007.

[20] F. D'Agostino, C. Gennarelli, G. Riccio, and C. Savarese, "Theoretical foundations of near-field-far-field transformations with spiral scannings," Prog. in Electromagn. Res., vol. PIER 61, pp. 193-214, 2006.

[21] O.M. Bucci, G. D’Elia, and M.D. Migliore, "A new strategy to reduce the truncation error in near-field / far-field transformations," Radio Sci., vol. 35, pp 3-17, 2000.

[22] F. D'Agostino, F. Ferrara, C. Gennarelli, R. Guerriero, and G. Riccio, "An effective technique for reducing the truncation error in the near-field-far-field transformation with plane-polar scanning," Prog. in Electromagn. Res., vol. PIER-73, pp. 213-238, 2007.

[23] F. D'Agostino, F. Ferrara, C. Gennarelli, R. Guerriero, G. Riccio, and C. Savarese, "An efficient technique to lower the error due to the truncation of the scanning region in a bi-polar facility," Microw. Opt. Technol. Lett., vol. 49, pp. 3033-3037, December 2007.

[24] P.C. Hansen, Rank-deficient and discrete ill-posed problems, SIAM, Philadelphia, USA, 1998.

[25] S.L. Belousov, Tables of normalized associated Legendre polynomials. Pergamon Press, Oxford, 1962. 